\title{
marges Marges
}

revue d'art contemporain Revue d'art contemporain

$17 \mid 2013$

Remake, reprise, répétition

\section{Archéologie d'un scandale : RoS Indexical d'Yvonne Rainer ou Le Sacre du printemps rejoué}

Archaeology of a scandal : RoS Indexical from Yvonne Rainer or The Rite of

Spring re-enacted

Laurence Corbel

\section{OpenEdition}

\section{Journals}

Édition électronique

URL : http://journals.openedition.org/marges/102

DOI : $10.4000 /$ marges. 102

ISSN : 2416-8742

\section{Éditeur}

Presses universitaires de Vincennes

Édition imprimée

Date de publication : 1 novembre 2013

Pagination : $10-20$

ISBN : 978-2-84292-394-5

ISSN : $1767-7114$

\section{Référence électronique}

Laurence Corbel, «Archéologie d'un scandale : RoS Indexical d'Yvonne Rainer ou Le Sacre du printemps rejoué », Marges [En ligne], 17 | 2013, mis en ligne le 01 novembre 2014, consulté le 05 mai 2019. URL: http://journals.openedition.org/marges/102 ; DOI : 10.4000/marges.102 


\section{Archéologie d'un scandale: RoS Indexical d'Yvonne Rainer ou Le Sacre du printemps rejoué}

/ 1 Il en reste un film de Babette Mangolte qui a servi de support à cette analyse. Je remercie Michèle Bargues et Evelyne Boutevin du Centre Pompidou de m'avoir permis de visionner ce film.
Le Sacre du printemps provoqua lors de sa création à Paris en 1913, il y a presque un siècle, un véritable scandale: né d'une collaboration entre le compositeur Igor Stravinsky et le chorégraphe Vaslav Nijinsky, cette œuvre a inspiré de nombreux chorégraphes et suscité plus de cent reprises. Celle que présente Yvonne Rainer en 2007/1, sous le titre RoS Indexical (Rite of Spring Indexical), est un hommage iconoclaste rendu à une œuvre majeure du répertoire chorégraphique du $20^{\mathrm{e}}$ siècle. Comment qualifier cet hommage? S'agit-il d'une reprise, d'une adaptation, d'une interprétation ou d'une révision? En dépit de la proximité sémantique de ces termes et au-delà de la simple querelle de mots, il est utile de définir le statut de cette pièce chorégraphique: si elle comprend de nombreuses références au Sacre, celles-ci relèvent autant du registre de la célébration que de celui de la parodie et de la déconstruction. Au regard des nombreuses reprises que compte ce ballet reconnu comme l'un des chefs d'œuvre de la modernité, la pièce chorégraphique de Rainer est singulière: non seulement parce qu'elle interroge le statut de la reprise dans une perspective à la fois diachronique et synchronique mais aussi parce qu'elle articule ses différentes formes. 
Reprise atypique mais exemplaire, elle mérite d’être étudiée à plusieurs titres:

1/ En premier lieu, RoS Indexical fait référence à d'autres reprises du Sacre du printemps. Elle intègre ainsi les différents modes de reprise d'une œuvre chorégraphique: de la reconstitution à l'interprétation en passant par la réécriture ou l'adaptation et la révision - au sens que ce terme prend chez les historiens. La pièce de Rainer n'est donc pas simplement une reprise de plus dans la longue histoire du Sacre mais elle propose une réflexion sur ce geste artistique, ses enjeux et ses finalités, en particulier pour cet art éphémère qu'est la danse. 2/ RoS Indexical, qui décline différentes modalités de la reprise, se distingue par sa dimension polyphonique: de la référence, voire de la révérence, à la parodie en passant par l'interprétation, cette œuvre joue de l'appropriation de certains éléments du répertoire du Sacre du printemps. Celui-ci s'est constitué au fil de l'histoire, travaillant et traversant l'épaisseur historique de cette œuvre, la suite des reprises le prolongent et le mettent en perspective.

3/ Il s'agit en outre, comme on l'expliquera, d'une reprise sans original. En effet, il ne subsiste du Sacre du printemps qu'une mémoire partielle. L'histoire de cette pièce chorégraphique invite ainsi à repenser le rapport de la reprise à son modèle: plutôt qu'elle n'hérite d'un passé, la reprise construit ici sa propre généalogie.

4/ Enfin, le titre (RoS Indexical) place cette pièce sous le signe de l'indexicalité, soulignant la dimension déictique et contextuelle de cette reprise. Indexer le Sacre, c'est lui agréger du sens, des significations en fonction d'un contexte. Quel est ce contexte? Une commande pour l'édition 2007 de Performa, biennale organisée par l'historienne d'art, critique et commissaire RoseLee Goldberg depuis 2005 à New York autour de la performance/2; un moment dans le parcours de Rainer qui, après avoir délaissé la danse pour le cinéma entre 1975 et 2000, a repris l'écriture chorégraphique. Le contexte est aussi celui du temps de la création de RoS Indexical, une étape dans l'histoire du Sacre et de ses nombreuses reprises. Il s'agira ainsi de confronter le geste artistique de la reprise au principe de l'indexicalité qui sous-tend le travail de Rainer. La notion d'index renvoie-t-elle à une conception spécifique de la reprise et des opérations qu'elle met en jeu?

\section{Un hommage critique}

Une salle debout qui applaudit, hurle ou siffle, des danseurs égarés, un chef d'orchestre qui essaie de garder la cadence malgré le tumulte, une presse déchainée... Du Sacre, on conserve d'abord le
/ 2 La pièce a été présentée deux fois dans ce cadre au Hudson Theater puis dans plusieurs villes européennes (Berlin, Dublin, Marseille, Paris, etc.). 
/3 François Lesure précise que le scandale fut en partie organisé: « Le 29 mai, pour la première, Diaghilev avait crubon d'organiser une sorte de claque: des jeunes loups, installés derrière les corbeilles, qui en fait allaient largement contribuer à déclencher le tumulte dans la salle. Peu après le début de l'introduction, on entendit déjà quelques rires et moqueries, qui se renouvelèrent lorsque le rideau se leva sur les danseurs.

C'est alors que la petite claque de Diaghilev, protesta, voulant les faire taire, avec assez de véhémence pour créer le vacarme. ». François Lesure « L'accueil parisien du sacre », dans Les Carnets du Théâtre des Champs Elysées, Paris, Cicero, 1990, p. 15.

/4 Voir Shelly C. Berg, Le Sacre du printemps: Seven Productions from Nijinsky to Martha Graham qui étudie I'histoire du ballet à travers Leonide Massine (1920, 1930), Maurice Béjart (1959), Paul Taylor (1980), Richard Alston (1981), and Martha Graham (1983).

/5 Millicent Hodson, «Puzzles chorégraphiques. Reconstitutions du Sacre de Nijinsky ", dans Les Carnets du Théâtre des Champs Elysées, op. cit., p. 45.

/6 Le ballet fut filmé en 1989 et diffusé sur Arte dans le cadre souvenir d'un scandale: la première du ballet présentée le 29 mai 1913 à Paris, au Théâtre des Champs Elysées, se déroule dans une ambiance si chaotique que Nijinsky est obligé de scander les rythmes de la partition de la coulisse pour les danseurs qui n'entendent plus la musique/3. La critique exprime presque unanimement son désaveu face à cette œuvre qui perturbe les convenances musicales et les normes du ballet: sa danse rituelle provocante aux postures novatrices, la rythmique musicale saccadée et l'étrangeté des costumes lui valent le qualificatif de «massacre du printemps ». Avec la réception houleuse et controversée de cette œuvre fondatrice considérée comme "symbole de la modernité ", l'absence d'images et d'archives filmées, la postérité chorégraphique/4 du Sacre ont également contribué à en faire une œuvre mythique. Mais c'est avant tout la perte des notations de Nijinsky qui a façonné de manière décisive la postérité de ce ballet. S’il existe une version « historique » du Sacre, c'est sous la forme de la reconstitution issue des recherches menées pendant de longues années par Millicent Hodson: "Rassembler les fragments perdus ou éparpillés de la chorégraphie de Vaslav Nijinsky pour Le Sacre du printemps, expliquet-elle, fut comme de travailler à la reconstruction d'un grand puzzle. Toutes les pièces, avec leurs découpes bizarres, leurs couleurs et leurs irrégularités de métrique, devaient être réinsérées dans ce grand tableau “de la Russie païenne en deux actes"/5. ». C'est sur la base de cette reconstitution fondée sur les récits de protagonistes ou de témoins - notes de Stravinsky en marge de la partition, croquis de la peintre Valentine Cross-Hugo, critique de l'époque, notes léguées par Marie Rambert qui fut l'assistante de Nijinsky - ainsi que des photographies et dessins, que la version du Joffrey Ballet - des représentations qui furent données à partir de 1987-a été réalisée, version dont on a tiré un film documentaire/6 et à partir de laquelle un film de fiction Riot at the Rite a été tourné par Andy Wilson en 2005 pour la BBC.

Nombreux sont les chorégraphes/7 qui ont eu le désir de s'affronter au Sacre: leurs reprises couvrent un large spectre de styles chorégraphiques et des approches très différentes de la partition musicale ou chorégraphique et du thème. Si Rainer ne se situe pas dans la perspective d'une reprise historique, elle ne prend pas pour autant le parti d'une re-création totale de l'œuvre comme l'ont fait Maurice Béjart, Anjelin Preljocaj ou Pina Bausch: son travail se situe dans le double registre de l'hommage et de la critique. Une telle position ne manque pas de surprendre chez une chorégraphe, dont les principes chorégraphiques défendus depuis les années soixante conduiraient plutôt à contourner la forme du ballet. Ses pièces les 
plus connues, comme Parts of Some Sextets (1965) ou Trio A (1966), s'écartent des éléments, comme la dimension narrative, la présence de personnages ainsi que la théâtralité, qui définissent traditionnellement le ballet. Si elle rompt avec le style académique des ballets qui développent une danse expressive (avec ballerines sur pointe et en tutu), la chorégraphie du Sacre reste néanmoins prise dans le cadre traditionnel de la double dépendance de l'intrigue et de la musique dont la danse s'affranchira progressivement. A priori, tout semble donc opposer Le Sacre - sa théâtralité, son expressivité, son spectacle, ses personnages - et la danse dite "postmoderne » et son esthétique du refus/8 que Rainer a présentée dans son célèbre manifeste négatif: "Non au grand spectacle non à la virtuosité non aux transformations et à la magie et au faire-semblant non au glamour et à la transcendance de l'image de la vedette non à l'héroïque non à l'anti-héroïque non à la camelote visuelle non à l'implication de l'exécutant ou du spectateur non au style non au kitsch non à la séduction du spectateur par les ruses du danseur non à l'excentricité, non au fait d'émouvoir ou d'être ému/9. ».

\section{L'archéologie d'un ballet ou la construction d'une mémoire feuilletée}

De fait, la chorégraphie de RoS Indexical n'a avec Le Sacre, du moins ce que l'on connaît de la version historique, que des rapports assez lointains. Au corps de ballet des quarante-six danseurs présents dans la version historique, Rainer substitue quatre interprètes féminines/10, au décor imposant de Nicolas Roerich un décor minimaliste constitué d'un simple canapé et de pancartes de mots qui flottent dans l'espace scénique, à la partition originale de Stravinsky, la bande son extraite du film réalisé par Andy Wilson. Restent les costumes des danseuses qui évoquent ceux de Roerich, certains pas de la chorégraphie originale du Sacre aisément identifiables et la musique reconnaissable en dépit des cris de protestation des spectateurs qui la recouvrent. Interrompue à plusieurs reprises par des temps de récupération pendant lesquels les interprètes s'assoient sur le canapé, la chorégraphie de Nijinsky est redistribuée dans des séquences interrompues par des solos - pantomimes ou pas de danses contemporaines (rock, salsa) - qui prennent l'allure d'improvisations ou encore par des incidents inattendus (l'irruption sur scène de spectateurs protestataires). Si la chorégraphie de RoS se présente, au premier abord, davantage comme une succession aléatoire de mouvements que comme partition organisée, elle se révèle, du moins à l'œil averti, comme une œuvre palimpseste composée d'une soirée

thématique sur Le Sacre du printemps.

/7 Parmi lesquels Lester Horton (1937), Mary Wigman (1957), Maurice Béjart (1959), Pina Bausch (1975), Martha Graham (1984), Jérôme Bel (1995), Anjelin Preljocaj (2001), Raimund Hogue (2004), Lorenzo de Brabandere (2004) et Jean-Claude Gallota (2011). Plusieurs reprises sont programmées en 2013 pour le centenaire de la création: notamment Sacre \# 197 de Dominique Brun au Centre national de la danse et Le Sucre du printemps de Rachel Garcia et Marion Musac à Chaillot. À ces pièces chorégraphiques, il faut ajouter l'installation vidéo The Rite of Spring de Katarzyna Kozyra (1999-2002).

/8 Sally Banes, "Yvonne Rainer: l'esthétique du refus ", dans Terpsichore en baskets. Post-modern dance, trad. D. Luccioni, PantinParis, CND-Éditions Chiron, 2002, p. 113.

/9 No Manifesto (1965) d'Yvonne Rainer, repris dans Terpsichore en baskets. Post-modern dance, op. cit., p. 90.

/10 Il s'agit des mêmes interprètes que pour la reprise d'Agon de George Balanchine : Pat Caterson, Sally Silvers, Emily Coates, Patricia Hoffbauer. Ces danseuses, dont l'âge, le physique 
et la formation professionnelle (classique ou contemporaine) diffèrent, ont déjà collaboré avec Rainer en 2006 pour la reprise d'Agon de Balanchine. La chorégraphe, suivant la tradition du travail collectif prôné dans Grand Union, a d'ailleurs sollicité leurs conseils et leurs compétences spécifiques pendant les répétitions de RoS.

/11 Yvonne Rainer, «1000 words: Yvonne Rainer Talks about RoS Indexical, 2007 ", Artforum, XLVI, $\mathrm{n}^{\circ} 3$, novembre 2007, p. 328.

/12 « Finalement, j'ai fini par penser à RoS Indexical à la fois comme de la pédagogie et comme du loisir, comme une sorte de vaudeville pédagogique qui intègre des traces, une analyse et un hommage.". Voir Yvonne Rainer, « RoS Indexical » dans RoseLee Goldberg (sld), Every where and All at Once: An Anthology of Writings on Performa 07, Zurich, New York, 2009, p. 108.

/13 On emploie ici ce terme dans son sens courant, sans référence à celui qu'il prend lorsqu'il désigne certaines stratégies artistiques, par exemple celles auxquelles Douglas Crimp s'est intéressé.

/14 Précisons que certaines reprises tendent à devenir des œuvres en soi dont on propose ensuite de différentes strates temporelles qui sont autant des jalons dans la reconstitution de la mémoire de cette pièce que des éléments destinés à la contextualiser.

C'est d'abord la radicalité du Sacre qui intéresse Rainer: ce ballet représente, en effet, un tournant dans l'histoire de la danse, une véritable rupture au regard des règles du jeu chorégraphique. Sous cet aspect, on ne manquera pas de relever des phénomènes d'échos entre Le Sacre et RoS Indexical: la chorégraphie de Nijinsky est au ballet classique ce que RoS Indexical est au ballet moderne. Si le jeune prodige des ballets russes et la chorégraphe postmoderne s'opposent dans les principes qu'ils mettent en œuvre, ils partagent une même volonté de rupture avec la tradition chorégraphique de leur temps. Par-delà les différences de style ou d'interprétation, bon nombre de reprises du Sacre ont suscité chez le public un même refus d'intégrer dans le champ de la danse des chorégraphies que l'on considère indignes de l'être. Comme Nijinsky, Rainer a voulu élargir les frontières dans lesquelles on cantonnait la danse: en y intégrant des mouvements ordinaires comme la marche ou la course, elle prolonge et radicalise la démarche du chorégraphe russe qui a, pour sa part, introduit des postures qui rompent avec la conception académique de la grâce (pointe des pieds en dedans, têtes penchées, épaules décalées, corps courbés, petits sauts répétitifs, piétinements opposés à l'élévation prisée par la danse classique). Plus encore, ces reprises relèvent de la démarche d'appropriation caractéristique de la danse postmoderne quand elle s'empare des gestes ordinaires (marcher, courir, ramper, se coucher, etc.).

Prolongement des avant-gardes, la danse postmoderne, dont Rainer est une des figures pionnières, poursuit le travail de sape amorcé par Nijinsky pour renverser le cadre classique de la danse. Mais si elle se situe assurément dans le sillage de la danse postmoderne des années soixante, RoS Indexical y apporte quelques infléchissements : aux mouvements de la vie ordinaire, Rainer ajoute les gestes et expressions visuelles empruntés à deux acteurs - Sarah Bernhardt et William Robins, un " génie cinétique/11 » selon Rainer - élargissant ainsi encore le champ de la danse aux mimiques burlesques et au jeu mélodramatique. Tout en poursuivant ainsi le processus de «dé-définition » de la danse, Rainer comble également les blancs de la mémoire partielle du ballet et renouvelle avec une pointe d'humour, les principes peut-être austères d'inexpressivité et de neutralité de la danse qualifiée de "postmoderne » : sa reprise est à la fois intensification des orientations impulsées par la chorégraphie novatrice de Nijinsky dont elle retourne au passage quelques principes et prise de distance avec sa propre pratique chorégraphique dont elle 
perturbe ici l'orthodoxie. Qualifiant cette pièce de « vaudeville pédagogique/12 », Rainer en souligne aussi la double ambition: divertir, éduquer, autrement dit redéfinir les espaces de la danse et remettre en cause notre regard.

La reprise de Rainer se situe donc dans un mouvement d'appropriation/13 et de déconstruction. D’une part, elle choisit de travailler non à partir de la musique originale de Stravinsky mais, dans le sillage de la reconstruction sur la bande son de la version filmée du Sacre. Toutefois, ce n'est pas la version filmée de cette reconstitution qu'elle utilise mais le film tourné pour la BBC, Riot at the Rite [Émeute au Printemps] (2005), dont elle reprend l'extrait sonore, qui correspond à la première du spectacle au Théâtre des Champs Elysées où la musique de Stravinsky est recouverte par la cacophonie du public. Rainer indexe ainsi la part scandaleuse du Sacre; par le choix de cette bande son, elle donne à entendre ce que fut la réception de ce ballet lors de sa première présentation où l'on vit la salle protester avec violence. Elle ne cherche pas tant à pérenniser la réputation scandaleuse du Sacre qu'à rappeler que celle-ci est désormais indissociable de l'identité de l'œuvre. Devenu un monument de l'histoire de la danse, en partie par la force de ce scandale, Le Sacre est d'abord identifié à la réputation sulfureuse que lui valut cette première qu'on dit être la plus scandaleuse dans l'histoire des arts. Or, le scandale occulte autant l'œuvre qu'il lui donne de visibilité, surtout si l'on s'avise que l'histoire des reprises du Sacre a largement contribué à figer cette image au détriment de son caractère radical et novateur, en mettant en avant la dimension spectaculaire de la rupture plutôt que sa teneur critique. Il s'agit ainsi de faire un sort aux effets de mythification qui touchent à la représentation que l'on a aujourd'hui de cette œuvre.

Rainer inscrit donc RoS dans une généalogie du Sacre qui relie certaines des reprises réalisées par des chorégraphes célèbres/14, la reconstitution de la version " historique » à laquelle se greffent des éléments extérieurs à l'histoire de ce ballet: des extraits de Trio A une des pièces les plus célèbres de la chorégraphe -, les mimiques du comique américain Robin Williams et le jeu corporel de Sarah Bernhardt dans une pièce du répertoire classique.

À rebours du processus de mythification qui met l'histoire entre parenthèses, Rainer propose une représentation stratifiée, un agencement de reprises et de temporalités hétérogènes - les avant-gardes dont le Sacre est une illustration, les années soixante où se développe la danse postmoderne, le début du $21^{\mathrm{e}}$ siècle, moment de la création de cette reprise - et montre ainsi comment se constitue la mémoire d'un spectacle. Au fil de la pièce, on repère des traces de des « reprises » : c'est le cas de la version de Maurice Béjart présentée en 1959 et dont on a donné une reprise en 2004, puis en 2005 , et de celle de Pina Bausch créée en 1975 et reprise en 2010. Il y a ainsi le Sacre de Maurice Béjart, le Sacre de Pina Bausch: si ces chorégraphes ont conservé le titre original de la pièce, leurs versions sont présentées comme des « re-

créations ». Chacune est devenue une œuvre en soi, comme le montre cette déclaration de Gil Roman lors de la reprogrammation du Sacre de Béjart en 2005 . Quand on lui demande le degré de fidélité à l'œuvre de Béjart, il rétorque sans équivoque: «Pas question de changer une virgule, pas un pas ni un costume décidé par Maurice Béjart » avant d'ajouter: «Mais la danse n'a rien d'abstrait, elle est faite de corps et d'esprits. De chaque interprète émane quelque chose d'intimement différent, et c'est tant mieux. Il ne faut pas que Le Sacre devienne une pièce de musée, il faut continuer à lui insuffler vie et vérité. C'est là le seul changement: l'âme de ses interprètes. ", cité par Anne-Sylvie Sprenger, L'Hebdo, 9 juin 2011, p. 76. 
/15 Rappelons que par cetermed'" archéologie », Foucault désigne les « analyses qui font apparaître à la fois les diverses formes de succession qui se superposent dans le discours (et par formes, il ne faut pas entendre simplement les rythmes ou les causes, mais bien les séries elles-mêmes), et la manière dont s'articulent les successions ainsi spécifiées. ". Michel Foucault, L'Archéologie du savoir (1969), Paris, Tel/Gallimard, 2008, p. 230.

/16 On pourra se reporter aux travaux sémiotiques de Peirce qui définit trois types de signes: l'icône (signe par ressemblance), le symbole (signe par convention) et l'index (signe par connexion physique). Voir Charles Sanders Peirce, Écrits surle signe, trad.

G. Deledalle, Paris, Seuil, 1978. versions antérieures du Sacre (parmi les spectateurs qui font irruption sur la scène au beau milieu du spectacle, l'un porte un costume ressemblant à ceux des danseurs du Joffrey Ballet) ou de chorégraphies de Rainer (Trio $A$ dont le spectateur averti peut reconnaître un passage, la présence du canapé qui fait écho à l'utilisation de nombreux objets fonctionnels dans les chorégraphies des années 1960-1970). Adoptant un point de vue archéologique, proche de la démarche que Foucault a construite/15, Rainer réorganise dans une chorégraphie polyphonique et hétérogène les différentes strates qui construisent l'histoire de ce ballet et qui ont forgé sa dimension mythique, en privilégiant certains éléments - les plus spectaculaires - au détriment de ceux, moins anecdotiques et plus souterrains, où se loge pourtant la force créatrice de cette chorégraphie. De l'un à l'autre de ces emprunts, une dialectique se construit par le retour du passé dans le présent et par l'incursion du présent dans le passé, l'histoire de la danse se repliant sur elle-même en chambre d'échos. Si la chorégraphie de Rainer semble à première vue très éloignée de la reconstitution de la version originale du Sacre, elle lui est pourtant plus « fidèle » que d'autres versions - celles de Béjart, de Bausch, de Preljocaj par exemple - qui en conservent le titre et suivent la thématique de la pièce tout en faisant l'impasse sur la version historique au profit de véritables re-créations. Rainer propose, quant à elle, une lecture à plusieurs niveaux où s'entremêlent, comme on l'a vu, des emprunts à la chorégraphie d'origine - certains pas et figures de la chorégraphie reconstituée par Millicent Hodson et Kenneth Archer en 1987 -, quelques motifs des costumes de la scénographie originale de Roerich, la bande sonore du film de la BBC Riot at the Rite - et des éléments ajoutés - tels que les références au jeu de Sarah Bernhardt et de Robin Williams donnant une tonalité burlesque à cette pièce.

\section{Décadrer, indexer, montrer}

Ces différents agencements opèrent ainsi un double décadrage, spatial et temporel: le premier questionne l'espace de la représentation en effaçant le partage traditionnel entre la scène et le public que l'on voit sur scène et que l'on entend sur la bande son tandis que le second télescope des registres artistiques hétérogènes (dramatique, burlesque, expressif) tissant également des liens entre des temps divers.

Le principe de l'indexicalité ou plus exactement la pratique de l'index, qui est ici appliquée au Sacre, définit le statut de cette pièce chorégraphique qui fonctionne comme un vecteur d'attention/16, un doigt pointé, dirigé sur tous les éléments qui y sont disposés selon 
un agencement précis. De la bande son au décor, de la chorégraphie aux costumes, de la scénographie à la performance des quatre danseuses, Rainer travaille avec et sur les traces et les archives du Sacre: elle propose non pas stricto sensu une représentation mais une mise en abyme des fragments de certaines de ses représentations passées, qui fait circuler le sens entre différents médiums filmiques, chorégraphiques, dramatiques. C'est encore cette fonction d'index qui est dévolue aux pancartes, qui apparaissent sur la scène au milieu du spectacle. $Y$ sont inscrits au recto et au verso trentedeux mots qui listent les aspects significatifs du Sacre: "suffer», "glories », "terror», " decay », "savage », "act», "lapsed », « if not now, when? », "who? me? », "sofa», "lunch», "aargh », etc. Partie prenante du décor, ils constituent aussi une sorte de glossaire qui privilégie certaines composantes du spectacle tout en occultant d'autres aspects de l'intrigue du Sacre: Rainer explique qu'elle a voulu éviter les " histoire ", "tribu », "sacrifice », etc., avec leurs références explicites aux rituels païens imaginés par l'artiste Nicolas Roerich lors de sa collaboration avec Nijinsky et Stravinsky pour la version originale du Sacre. Une stratégie plus oblique est indiquée, un mouvement ambigu d'allers-retours dans le temps, qui va de la présence émotionnelle de "terreur» à la banalité de « repas » et « lutte », et "sinon maintenant, quand? » ajoutés pour faire bonne mesure/17.

Il s'agit ainsi moins de proposer une interprétation, d'apporter un commentaire ou de donner des clés de lecture de l'œuvre comme le font souvent les reprises que d'inviter le public à mettre en relation, en fonction de ses connaissances et de ses expériences, les strates qui composent l'histoire du Sacre. À charge pour les spectateurs de constituer des réseaux de sens en relation avec ce qu'ils voient sur scène, ce qu'ils entendent et ce qu'ils connaissent du Sacre.

L'envers de cette pratique de l'index ou son corrélat, c'est le refus du jeu référentiel de la chorégraphie: est ici reconduit le principe central de la danse postmoderne selon lequel les interprètes n'imitent pas des gestes ou des expressions mais les montrent ou les citent: à propos des mouvements présentés dans Trio $A$, Rainer explique qu'« ils ne sont pas mimétiques/18 »; ainsi «[...] ce que fait quelqu'un est plus important que l'exhibition de personnages; [...] ainsi dans l'idéal, quelqu'un n'est plus soi-même, il n'est plus que "celui qui fait" de façon neutre/19 ». Les interprètes effectuent donc des gestes sans les incarner. Comme l'acteur chez Brecht qui est un « montreur », les danseuses de RoS ne s'identifient à aucun moment avec leurs personnages (la jeune vierge sacrifiée, Sarah Bernhardt ou Robin Williams), elles ne font que les citer.
/17 Yvonne Rainer, "RoS Indexical ", dans RoseLee Goldberg (sld), op. cit., p. 107.

/18 Yvonne Rainer, "La pensée musculaire", dans Le travail de l'art, $\mathrm{n}^{\circ}{ }_{1}$, Paris, automne/ hiver 1997, p. 97.

/19 ibid., p. 93. 
/20 Voir « RoS

Indexical », dans

RoseLee Goldberg (sld),

op. cit., p. 106.

/21 Yves Bonnefoy, "Lever les yeux de son livre », dans Nouvelle Revue de Psychanalyse, $\mathrm{n}^{\circ} 37$, printemps 1988 , p. 14 .

/22 Voir Luigi Pareyson, Esthétique. Théorie de la formativité, trad. G. Tiberghien et R. di Lorenzo, Paris, Éditions de l'ENS, 2007.
Cet effet de distanciation, lié à l'indexation, se trouve encore mis en œuvre lorsque Rainer fait venir une groupe de spectateurs sur la scène ou encore lorsqu'elle rend visible le travail de répétition, d'entraînement qui précède la représentation. La (re)présentation nous donne ainsi à voir ce qui est d'habitude relégué dans les coulisses du spectacle: les temps de pause que les danseuses s'octroient en allant s'asseoir sur le canapé ou la scène d'ouverture montrant les danseuses assises autour d'une table, des écouteurs aux oreilles, en train de fredonner l'ouverture du Sacre et de battre le rythme.

\section{Refaire, défaire et re-voir}

À propos de RoS Indexical, Rainer a déclaré qu'il n'est ni une «reconstitution » du Sacre comme c'est le cas pour la version du Joffrey Ballet, ni un "remake», ni une «interprétation». Si elle emploie parfois le terme d'« appropriation », c'est le terme de "revision/20 » qu'elle convoque le plus souvent: un terme que l'on peut entendre d'abord comme re-vision, comme seconde vision et aussi comme révision, au sens de mise à distance critique et de mise en question des sources que les historiens donnent à ce terme. Ce qui se joue dans la reprise, c'est en effet la question de la distance temporelle, de l'écart historique qui sépare le moment de la création du Sacre de ses reprises: au-delà de l'intérêt historique de la reconstitution d'un modèle ou d'un original supposé, c'est aussi dans le mouvement de lecture rétroactive, dans les écarts et les déplacements produits entre le point d'origine et sa réactualisation que réside l'intérêt de la reprise. Dans une telle perspective, une œuvre n'est vraiment compréhensible que si on entend le système d'échos qu'elle entretient avec ses reprises: comme le dit Yves Bonnefoy, « une grande œuvre est bien moins la réussite d'une personne que l'occasion qu'elle donne à d'autres de recommencer la recherche/21 ».

Contre la reprise-reconstitution conçue comme quête d'un original, d'une source où viendrait se loger la vérité de l'œuvre, Rainer choisit la démultiplication d'un sens disséminé dans les différentes occurrences de sa présentation. À la reprise-interprétation, elle préfère le montage ou l'agencement, la transposition qui ouvre des possibilités de variation et d'invention. Projeter dans le présent une œuvre du passé que l'on actualise, voire que l'on « rajeunit » faute de quoi elle s'expose à n'être que la trace dépassée d'une époque, c'est activer ou réactiver sa "puissance formative/22 ». La reprise de Rainer n'est pas, on l'a compris, répétition ni simple recontextualisation mais agencement, reconfiguration, recomposition d'éléments. Rainer « revoit » cette œuvre classique, autrement dit la « révise» 
non pas en nous ramenant à ce qu'elle fut (sa version historique) mais en en transformant le cadrage, en la transposant dans un autre contexte.

Sa reprise rend ainsi visibles certaines des virtualités de l'œuvre. À l'instar des reprises qui révèlent les œuvres comme des foyers de variations potentielles, celle de Rainer invite à réfléchir le rapport des œuvres au temps, à l'histoire et pose la question de la reprise comme contribution à la pérennité d'une œuvre. Reprendre Le Sacre, n'est-ce pas, en effet, travailler à contretemps, s'emparer d'une œuvre marquée par l'esprit de son temps, une œuvre qui non seulement a fait date mais qui est aussi datée? Et la reprise ne permetelle pas de maintenir une œuvre du passé vivante, de la protéger du risque que sa valeur historique ne l'emporte sur ce que Riegl appelle sa "valeur d'actualité/23 »? Comment une œuvre peut-elle ne pas être réduite à la simple représentation d'« un moment déterminé de l'évolution dans un domaine quelconque de l'activité humaine/24 "? En d'autres termes, est-il possible de délier l'œuvre du moment de son apparition, de suspendre le temps de sa création, et à quelles conditions? Dans le cas précis du Sacre, outre la perpétuation de sa mémoire, ce sont le renversement du scandale en mythe et l'effacement de sa dimension subversive que Rainer met en jeu. Comment réactiver la part de provocation qui fut celle de ce ballet devenu une référence mythique?

Ces questions engagent le sens même du geste de la reprise: reprendre, est-ce entretenir une mémoire, rajeunir au sens d'actualiser, travailler le décalage temporel à partir duquel s'effectue la reprise, par nature différée? La reprise de Rainer ne répond pas tant au projet, qui s'expose toujours à une forme de pétrification, d'entretenir la mémoire d'une œuvre chorégraphique qu'à celui de produire une lecture du Sacre à partir du présent, de mettre ce mythe à l'épreuve du temps, de "réactiver » sa force critique en reconfigurant ses lignes de force.

Et ce n'est pas seulement la question de la possible « actualité » du Sacre qui est posée mais aussi, par ricochet, celle de son « invisibilité » originelle : comment expliquer que le Sacre ait provoqué pareil scandale lors de ses premières présentations (alors que l'on sait qu'un an après, lors de sa présentation à Londres, le spectacle fut acclamé), qu'il ait suscité chez les spectateurs un tel choc? Qu'est-ce qui fait donc la «visibilité » d'une œuvre? À quelles conditions une $œ u v r e$, une forme artistique devient ou redevient-elle visible? Comment comprendre la postérité de cette œuvre nécessairement marquée par son temps? Entre actualité et inscription dans l'histoire, le rapport au temps apparaît comme un enjeu central de la reprise.
/23 Aloïs Riegl, Le Culte moderne des monuments (1903), trad. J. Boulet, Paris, L'Harmattan, 2003, p. 91 et suiv.

/24 ibid., p. 81. 
/25 Gilles Deleuze et Félix Guattari, Qu'est-ce que la philosophie? (1991), Paris, Minuit, 2005 , p. 107.

/26 Gilles Deleuze, Proust et les signes, Paris, Presses Universitaires de France, 1964, p. 63.

/27 Jean-Philippe Antoine, «Statistique et métaphore ", dans Gabriel Tarde, Les Lois de l'imitation, Paris, Les Empêcheurs de tourner en rond, 2001, p. 29.

Si le temps a gommé une partie de la force provocatrice de la pièce, celle-ci conserve une puissance de subversion qu'il faut lui insuffler. La reprise montre ainsi qu'une œuvre n'est jamais achevée, que son sens reste suspendu aux lectures qu'on en fait autant qu'aux différentes réactualisations auxquelles elle donne lieu; plutôt que de limiter l'actualité d'une œuvre au seul présent, il faudrait alors la penser à l'aune de son devenir ainsi que le proposent Deleuze et Guattari à la suite de Foucault: "L'actuel n'est pas ce que nous sommes mais plutôt ce que nous devenons, ce que nous sommes en train de devenir, c'est-à-dire l'Autre, notre devenir-autre/25. " Dès lors, on comprend que l'actualité des œuvres interroge en même temps leur inactualité.

Traversée par des jeux de résonances, de diffractions, de variations, la reprise est « puissance de la différence/26 », productrice de sens. Elle entretient ainsi des liens étroits avec l'imitation telle que Gabriel Tarde la définit dans son célèbre texte Les Lois de l'imitation, comme lieu où se produit la différence: comme le dit Jean-Philippe Antoine dans sa préface, l'imitation est «le mouvement par lequel quelque chose se répète, et se répétant, se propage. Mais c'est aussi, et d'un seul tenant, le mouvement par lequel, se répétant, cette même chose se différencie, en quantité comme en qualité/27 ». Dans une telle perspective, la reprise ouvre des possibilités d'invention et de variation: le type de répétition qu'elle met en œuvre se traduit par un processus de différenciation qui est aussi celui d'une singularisation, ce que l'on peut appeler avec Tarde « invention » ou « découverte ».

La reprise est donc à la fois répétition et différence : répétition qui met à l'épreuve la portée artistique d'une œuvre, différence qui affirme une portée critique et subversive, les écarts et les déplacements permettant de mesurer le potentiel créateur, le «cœfficient artistique» de la pièce. Entre reconstruction et déconstruction, entre hommage et mise à distance, cette reprise de Rainer, qui se produit comme sa propre mise en question, est exemplaire du processus de réactualisations incessantes qui assure aux œuvres dites majeures une postérité sans les enfermer dans une forme canonique figée du dialogue que les reprises successives entretiennent non seulement avec leur modèle mais aussi les unes avec les autres.

\section{Laurence Corbel}

